\title{
Effects of changing alley color on the successive negative contrast effect
}

\author{
ELIZABETH D. CAPALDI \\ Purdue University, West Lafayette, Indiana 47907
}

\begin{abstract}
In Phase 1, four groups of rats received either large or small food reward in a black or white straight alley. In Phase 2 all rats received small food reward in the white alley. Rats that had received large reward in Phase 1 ran more slowly in Phase 2 to small reward than did rats that received small reward in both phases (successive negative contrast effect, or NCE). There was a temporary decrement in running speed for both groups that experienced a change in alley color, but the size of the NCE was not reduced by the change in alley color. It was suggested that the NCE can be used to measure the strength of connection between a particular cue and reward expectancies in instrumental learning situations. Within this context, the present results suggest that, under the conditions employed in this experiment, alley color was not strongly associated with reward expectancies.
\end{abstract}

When rats are shifted to small reward after a number of trials of receiving large reward, their speed temporarily drops below the level of a small reward control. This phenomenon is termed the successive negative contrast effect (NCE). In part the phenomenon has been attributed to negative effects (e.g., inhibition, frustration) on the instrumental response of receiving a reward less than expected (Black, 1968; Cleland, Williams, \& DiLollo, 1969). Recently, we showed that changing deprivation level up or down along with the decrease in reward size reduced or eliminated the NCE (Capaldi, Smith, \& White, 1977). And we interpreted these results to indicate that expectancies of reward become strongly associated with deprivation stimuli in instrumental learning situations. Within this analysis, changing deprivation along with a reduction in reward size reduces the animals' expectancy of receiving large reward, which reduces the negative effects of receiving small reward, thereby reducing the NCE.

Capaldi et al.'s (1977) results and interpretation suggest that the NCE can be used as a method of determining the strength of the connection between a given stimulus and reward expectancies. If changing a particular stimulus produces a large reduction in the NCE, this suggests that the particular stimulus was strongly associated with reward expectancies. And if changing a particular stimulus does not reduce the size of the NCE, this suggests that a particular stimulus was not strongly associated with reward expectancies.

In the present experiment this technique was employed to measure the strength of the connection between alley color and reward expectancies.

\section{METHOD}

\section{Subjects}

The subjects were 28 naive male rats, about 90 days old upon arrival from the Holtzman Company, Madison, Wisconsin.

\section{Apparatus}

The apparatus consisted of two adjacent alleys, one painted black and one painted white. Each alley measured $144.8 \mathrm{x}$ $8.9 \times 10.2 \mathrm{~cm}$, and the entire apparatus was covered with $1.3-\mathrm{cm}$ hardware cloth. The startbox of each alley was separated from the runway by a metal door which was dropped by pushing a button. Dropping the door started the first of three .01-sec clocks. Clock 1 was stopped (start time) and Clock 2 started when the rat interrupted a photobeam located $43.2 \mathrm{~cm}$ from the first. Interruption of the third photobeam stopped Clock 3 (goal time). The third photobeam was $30.5 \mathrm{~cm}$ beyond the second photobeam and $7.6 \mathrm{~cm}$ in front of an appropriately colored block of wood with a $1.9 \mathrm{~cm}$ deep x $3.17 \mathrm{~cm}$ diam hole used as a food cup. A manually lowered guillotine door, $34.3 \mathrm{~cm}$ from the rear of the goal compartment served to confine the rat to the goal area. Times from the three clocks were summed to produce total time.

\section{Procedure}

Rats were randomly assigned to four groups referred to by the reward magntitude experienced in Phase $1(\mathrm{~L}, 10.045 \mathrm{~g}$ Noyes pellets, or S, 1 .045-g pellet) and by whether the alley color in Phase 2 was different from or the same as that in Phase 1. Changed groups were run in the black alley in Phase 1 and the white alley in Phase 2; unchanged groups were run in the white alley in both phases. All rats received one pellet in Phase 2. A 12-g per day deprivation schedule began on Day 1. On Days 11,12 , and 13, each rat was handled for $90 \mathrm{sec}$ and fed 10 pellets or 1 pellet in its home cage (each rat was fed the number of pellets it was to receive in Phase 1). On Day 14 each rat received one trial and on Days 15-24 each rat received three trials each day, producing a total of 31 Phase 1 trials. In Phase 2 there were three trials per day for 8 days. Each rat was fed an amount each day sufficient to bring its total consumption to $12 \mathrm{~g}$ per day. Subjects were run in squads of four, consisting of one animal from each group. Each animal in a squad received Trial 1 in a random order, then each received Trial 2 in that order, etc., until that squad was done. Then the next squad was tested in the same manner. Each squad was fed its daily ration $15 \mathrm{~min}$ after trials were completed.

\section{RESULTS}

All times were converted to speeds $(1 / \mathrm{sec})$. Results in all alley sections were essentially the same, so only 


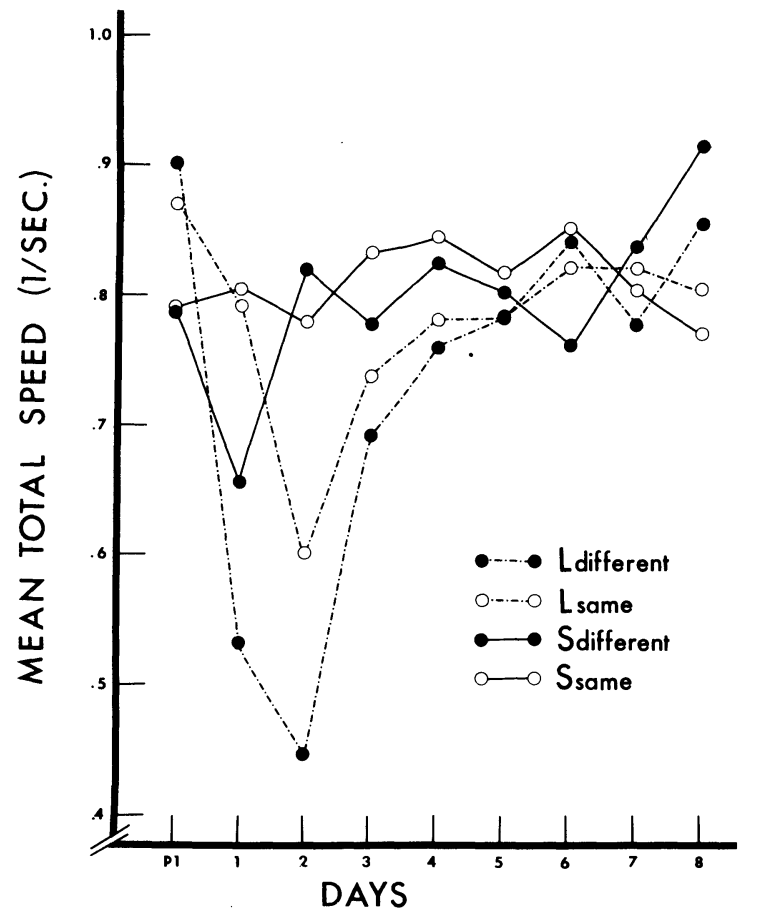

Figure 1. Mean total speed for each group on the last day of Phase 1 and each day of Phase 2.

total speeds are presented. In Phase 1 animals receiving 10 pellets ran faster than animals receiving 1 pellet $[F(1,23)=15.25, p<.005]$. Speeds did not differ significantly as a function of alley color $[\mathrm{F}(1,22)=1.31$, $\mathrm{p}>.20]$, nor did alley color and reward size interact significantly $(\mathrm{F}<1)$.

Figure 1 shows the mean speed for each group on the last day of Phase 1 and each day of Phase 2. As can be seen in Figure 1, animals that experienced a change in alley color dropped in speed below unchanged alley color groups on Day 1 of Phase 2, but the NCE occurred whether or not alley color was changed. Over all of Phase 2 the difference attributable to alley color in Phase 1 was not significant $[F(1,23)=1.15, p>.20]$ and animals shifted from $\mathrm{L}$ to $\mathrm{S}$ ran more slowly than those run only under $S[F(1,23)=4.22, p<.05]$. Most importantly, the Reward Magnitude by Alley Color interaction was not significant, either over all of Phase $2(\mathrm{~F}<1)$ or on any individual day. The Days by Alley Color interaction was significant; animals that experienced a change in alley color ran slower than unchanged groups on Day $1[\mathrm{~F}(1,23)=11.93, \mathrm{p}<.01]$ but not on Days 2.7 of Phase $2(\mathrm{Fs}<1)$.

\section{DISCUSSION}

The major finding obtained is that changing alley color produced a decrement in running speed but did not reduce the size of the NCE. Within the analysis offered by Capaldi et al. (1977), these results suggest that alley color was not very strongly associated with reward expectancies in this investigation. At the same time, changing alley color did produce a decrement in running speed, indicating the animals did notice alley color. It is certainly possible that with more preshift trials than employed here, changing alley color might have reduced the NCE, that is, alley color may become associated with reward expectancies late in training. And, of course, it is true that the animal can associate alley color with reward expectancies under certain experimental conditions, for example, in a differential conditioning situation where large reward is given in an alley of one color and small reward in an alley of another color with trials in the two alleys intermixed (e.g., Bower, 1961). In this situation it is widely assumed that expectancies of large reward and small reward are conditioned differentially to the two different alley colors. Support for this assumption is provided by the simultaneous NCE-slower running in the small reward alley by the differential conditioning group that receives large reward in the other alley than by a group that receives small reward in both alleys. Thus, perhaps, if a greater number of trials had been employed, changing alley color would have reduced the NCE. Or perhaps the procedure of intermixing trials of two different colors with two different associated reward magnitudes produces a stronger association between alley color and reward expectancies than the procedure employed here. Regardless of these considerations, the fact that changing alley color had no effect on the NCE here, while Capaldi et al. (1977) found that changing deprivation up or down following as few as 25 preshift trials eliminated the NCE, suggests that deprivation stimuli may be more strongly associated with reward expectancies than alley color in a simple straight alley situation, at least early in training.

\section{REFERENCES}

BLACK, R. W. Shifts in magnitude of reward and contrast effects in instrumental and selective learning: A reinterpretation. Psychological Review, 1968, 75, 114-126.

BOWER, G. H. A contrast effect in differential conditioning. Journal of Experimental Psychology, 1961, 62, 196-199.

Capaldi, E. D., Smith, N. S., \& White, L. A. Control of reward expectancies by drive stimuli. Journal of Experimental Psychology: Animal Behavior Processes, 1977, 3, 156-177.

Cleland, E. A., Williams, M. Y., \& Dilollo, V. Magnitude of negative contrast in relation to drive level. Psychonomic Science, 1969, 15, 121-122.

(Received for publication April 6, 1978.) 